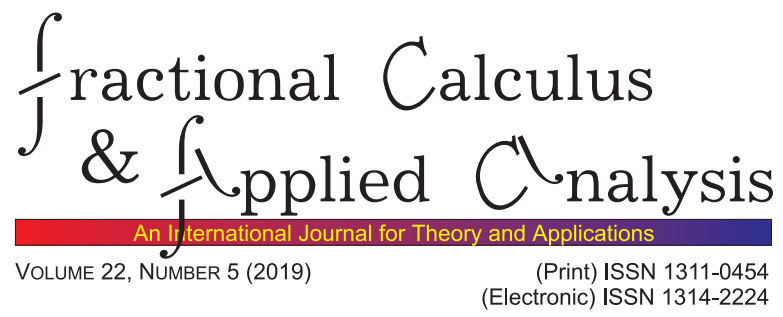

RESEARCH PAPER

\title{
IDENTIFICATION FOR CONTROL OF SUSPENDED OBJECTS IN NON-NEWTONIAN FLUIDS
}

\author{
Isabela Birs ${ }^{1,2,3}$, Cristina Muresan ${ }^{1}$, Dana Copot ${ }^{2,3}$, \\ Ioan Nascu ${ }^{1}$, Clara Ionescu ${ }^{2,3}$
}

\begin{abstract}
This paper proposes a framework for modelling velocity profiles and suspended objects in non-Newtonian fluid environment. A setup is proposed to allow mimicking blood properties and arterial to venous dynamic flow changes. Navier-Stokes relations are employed followed by fractional constitutive equations for velocity profiles and flow. The theoretical analysis is performed under assumptions of steady and pulsatile flow conditions, with incompressible properties. The fractional derivative model for velocity and friction drag effect upon a suspended object are determined. Experimental data from such an object is then recorded in real-time and identification of a fractional order model performed. The model is determined from step input changes during pulsatile flow for velocity in the direction of the flow. Further on, this model can be employed for controller design purposes for velocity and position in pulsatile non-Newtonian fluid flow.
\end{abstract}

MSC 2010: Primary 26A33; Secondary 76A05, 76D05, 93B30, 92C55

Key Words and Phrases: non-Newtonian fluid; pulsatile flow; drag force; fractional order impedance models; motion control; suspended object motion; step response; identification

\section{Introduction}

Autonomous object modeling and control in non-Newtonian fluid environment has a manifold of cross-disciplinary applications in the fields of

(c) 2019 Diogenes Co., Sofia

pp. 1378-1394, DOI: 10.1515/fca-2019-0072

DE GRUYTER 
biology, medicine, (nuclear) physics and fluid mechanics. Whether the purpose is to position particles in electro-magnetic actuated fluids (e.g. in steel manufacturing) [2], build a constitutive model for geomaterials [3] or guide a nano-sensor for detection of structural changes in arterial walls [20, 13, the necessity of apriori model for analysis is always justified. There are applications which require suspended object free motion, such as in ground water transportation [16, but also precise orientation and positioning is required via closed loop control, for instance in electromagnetic stirring of liquid steel velocities 9 .

Most properties of non-Newtonian fluids overlap with that of viscoelastic materials, such as polymers, lung tissue, gel substances, rubber, etc [20, 27, 28. Specific properties as memory, creep and shear stress do not follow classical Newton's law of viscosity and has been manifold proven to be well characterized by combinations of power-law and exponential functions [11. These are non-rational expressions of combined nonlinear effects in material creep and strain, and have been well characterized by the Mittag-Leffler function [18, 19, 13, 12.

To investigate the problems posed for modeling, identification and control objectives in such non-Newtonian environments, lab scale setups mimicking the main properties are very useful and accessible for research. This paper proposes such a lab scale setup, featuring non-Newtonian fluid motion and submerged objects. Physically-based rationale allows to investigate the development of a fractional derivative constitutive equation for the velocity gradient in non-Newtonian fluid. The space-dependent fractional derivative can also capture possible non-local effects of velocity on the non-Newtonian fluid flow. Identification of a fractional order dynamic model for velocity is performed from step response measured data. The model can be further used for controller design of position and velocity in non-Newtonian fluid flow.

The paper is structured as follows. The Section 2 introduces the materials and methods uses in this work, namely, the fractional flow dynamics are derived, followed by velocity and frictional head loss equations. Next, the setup description and details on hardware and software components are given. The section ends with considerations for identification step. Section 3 gives an overview of the obtained results, along with discussions and limitations of the current status. The concluding Section 4 summarizes the main outcome and proposes the next steps to be acquired in the project. Furthermore, a brief familiarization with fractional order calculus relevant for control purposes is presented in the Appendix section. 


\section{Materials and methods}

2.1. Fractional flow dynamics in Non-Newtonian fluids. Classical constitutive relationship for shear stress in terms of the velocity gradient can be expressed as [17]

$$
\tau=\mu \frac{d u}{d y}
$$

with $\tau$ the viscous shear stress, $\mu(\mathrm{kg} / \mathrm{m} \mathrm{s})$ the dynamic viscosity, $u$ the flow velocity $(\mathrm{m} / \mathrm{s})$ and $y(-)$ the radial direction in normalized form, i.e. $y=$ $r / R$ with $R(\mathrm{~m})$ the radius of the pipeline and $r(\mathrm{~m})$ the radial coordinate. This relation no longer holds for non-Newtonian fluids as blood, detergent, gel, plasma, etc.

Our previous work on fractionalising compartmental models for drug concentration gradients in blood and tissue has indicated that each gradient can be expressed in terms of its kernel and integral form of mass transfer [6, 10]. Consider the pipeline with our fluid divided in compartments of equal infinitesimal distance. The gradient is given by

$$
u(1)-u(0)=k \int_{0}^{1} M(\tau) d \tau,
$$

where the $M$ denote mass or molar amounts of material in the respective compartment, $k(1 / \mathrm{s})$ are rate constants. Each of the mass transfer integral includes a kernel, i.e.

$$
u(1)-u(0)=k \int_{0}^{1} K \cdot \tilde{M}(\tau) d \tau .
$$

In the classic theory case, the kernel is simply equal to one. By choosing the kernel in an appropriate form of power-law, we can then use RiemannLiouville fractional integrals or others. This power-law kernel has been formerly introduced in earlier studies of non-Newtonian materials and fluids [25, 15, 1]. For instance, using the kernel

$$
K(t, \tau)=\frac{(t-\tau)^{\alpha-1}}{\Gamma(\alpha)}
$$

with $0<\alpha<1$, we have for $\alpha=1$ the classical case since $K=1$. Keeping in mind the Riemann-Liouville fractional integral is

$$
{ }_{0} D_{t}^{-\alpha} M(t)=\int_{0}^{1} \frac{(t-\tau)^{\alpha-1}}{\Gamma(\alpha)} \tilde{M}(\tau) d \tau,
$$

where $D$ stands for an integral when the order is negative and for a derivative when the order is positive. Finally, we can write (2.3) as

$$
u(1)-u(0)=k_{0} D_{t}^{-\alpha} M(t) .
$$

The zero initial values are common in compartmental models, but if it is not zero, we can use the Caputo derivative, in the form 


$$
{ }_{0} D_{t}^{1-\alpha} M(t)={ }_{0}^{C} D_{t}^{1-\alpha} M(t)+\frac{M(0) t^{\alpha-1}}{\Gamma(\alpha)},
$$

where the superscript $\mathrm{C}$ on the left denotes a Caputo fractional derivative. Note the units of the rate constant are no longer $t i m e^{-1}$ but time $e^{-\alpha}$.

In 29] has been proposed a model to describe time-dependent flow in non-Newtonian fluids with similar initial conditions as the presented study:

$$
\tau(t)=\tau_{0}+a \cdot \lambda_{\beta} \frac{d^{\beta-1} \dot{\epsilon}}{d t^{\beta-1}},
$$

where $0 \leq \beta \leq 1, a$ and $\lambda$ are material constants, and $\epsilon(\mathrm{m})$ denotes strain. This is a generalization of (2.1) following the fractionalisation rationale. This has been used (in various forms) to model Maxwell elements in mechanical models of viscoelasticity [25, 15]. Further on, these mechanical models are then the basis for electrical model analogous [14, 1].

To address the non-locality problem of properties in non-Newtonian fluids, one may use (2.8), for $0<\alpha=\beta-1<2$. The physical basis for this non-uniform velocity gradient can be the non-uniformity of fluid particles (e.g. mixtures of solid and liquid particles), molecular interaction, biological and chemical effects. Using the fractional derivative definition from [29],

$$
\frac{d^{\alpha} u(y)}{d y^{\alpha}}=\frac{1}{\Gamma(n-\alpha)} \int_{0}^{y} \frac{u^{(n)}(\tau)}{(y-\tau)^{\alpha-n+1}} d \tau,
$$

with $n-1<\alpha \leq n$, and $n$ the smallest integer greater than the order $\alpha$, the relationship between velocity gradient and viscous shear stress is given by

$$
\tau=\left\{\begin{array}{c}
\tau_{0}+\mu I^{1-\alpha} \frac{d u}{d y}, 0<\alpha<1 \\
\tau_{0}+\mu \frac{d u}{d y}, \alpha=1 \\
\tau_{0}+\mu I^{2-\alpha} \frac{d}{d y} \frac{d u}{d y}, 1<\alpha<2
\end{array}\right\},
$$

where $I^{1-\alpha}$ and $I^{2-\alpha}$ represent the fractional integral

$$
I^{\gamma} f(y)=\frac{1}{\Gamma(\gamma)} \int_{0}^{y}(y-\tau)^{\gamma-1} f(\tau) d \tau, \gamma>0
$$

2.2. Velocity profile in pipeline. The following assumptions are made for this study, which are also in agreement with the real-life setup application for this work:

- the non-Newtonian fluid is incompressible, and

- the flow is laminar.

Consider a suspended object as in Fig. 1. The force balance relation in the flow direction is given by

$$
A\left(p_{1}-p_{2}+\rho g l \sin (\phi)\right)-\tau \cdot 2 \pi r l=0
$$




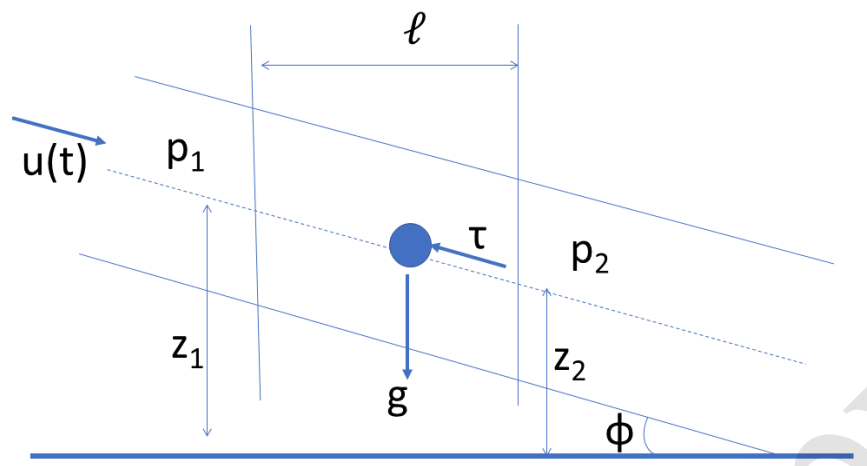

FiguRE 1. Schematic representation of a suspended object in pipeline.

with $A\left(\mathrm{~m}^{2}\right)$ cross section, $r(\mathrm{~m})$ radial direction, $\rho\left(\mathrm{kg} / \mathrm{m}^{3}\right)$ the density of the non-Newtonian fluid, and $\ell(\mathrm{m})$ is the length considered and $R(\mathrm{~m})$ the absolute radius of the cylinder. We have that $\sin (\phi)=\frac{z_{1}-z_{2}}{l}$, thus the hydraulic gradient is given by:

$$
J=\frac{\left(z_{1}+p_{1} / \rho g\right)-\left(z_{2}+p_{2} / \rho g\right)}{l}=\frac{2 \tau}{\rho g r} .
$$

Employing the velocity gradient constitutive equation from previous section we impose

and obtain

$$
\mu \frac{d^{\alpha} u}{d r^{\alpha}}=-\rho g r J / 2
$$

$$
u(r)-u(r=0)=-\frac{\rho g J r^{1+\alpha}}{2 \mu \Gamma(\alpha+2)} .
$$

Imposing the no slip condition, we have $u(r=R)=0$, hence:

$$
u(r)=\frac{\rho g J r^{1+\alpha}}{2 \mu \Gamma(\alpha+2)} .
$$

The velocity profile is written as

$$
u(r)=\frac{\rho g J}{2 \mu \Gamma(\alpha+2)}\left(R^{1+\alpha}-r^{1+\alpha}\right)
$$

which reduces to the Newtonian velocity profile for $\alpha=1$.

The maximum velocity is then calculated as

$$
u_{\max }=\frac{\rho g J R^{1+\alpha}}{2 \mu \Gamma(\alpha+2)}
$$


and the mean velocity is

$$
\bar{u}=\frac{\rho g J}{2 \mu \Gamma(\alpha+2)}\left(1-\frac{2}{3+\alpha}\right) R^{1+\alpha} .
$$

When the ration of these velocities is 0.5 , then we have Newtonian fluid - this is again the case for $\alpha=1$. So the velocity gradient may be nonuniform in non-Newtonian fluids.

2.3. Frictional head loss. For objects moving forward in a non-Newtonian fluid, we have a frictional head loss of velocity, which can be calculated from the mean velocity relation:

$$
F=\frac{2 \frac{\mu}{\rho} \Gamma(\alpha+2)}{g} \frac{3+\alpha}{1+\alpha} \frac{l \bar{u}}{R^{1+\alpha}}
$$

with $\frac{\mu}{\rho}$ the kinematic viscosity. This can be expressed in terms of the diameter of the cylinder $D=2 R$ :

$$
F=\frac{64}{\frac{2^{3-\alpha}}{(3+\alpha) \Gamma(1+\alpha)} \frac{\bar{u} D^{\alpha}}{\frac{\mu}{\rho}}} \frac{l}{D} \frac{\bar{u}^{2}}{2 g} .
$$

Again this reduces to the classical frictional head loss for Newtonian fluid when $\alpha=1$.

2.4. Lab scale setup description. An experimental setup was designed and implemented to mimic non-Newtonian fluid flow under pulsatile flow conditions. The rationale is to try to mimic pulsatile blood flow with nonNewtonian properties and further control suspended objects in it. Applications are of course related to nanorobot control for medical purposes, but other technical areas are also featuring similar modelling and control challenges. For instance, controlling liquids (e.g. liquid steel) by electromagnetic actuator force field onto regulating the flow profile and velocity in continuous casting.

The brain of the circulatory system is the LabVIEW ${ }^{T M}$ programmable myRIO ${ }^{T M}$ real-time controller. The myRIO ${ }^{T M}$ controls the variable flow pump CM10P7-1-24 which can generate a flow of up to 15 liters/minutes. The variable flow is realized with the pump driver EM-174A. The SNSFLOW201 flowmeter gives feedback regarding the actual flow of the liquid in the system. All components are supplied by a $24 \mathrm{~V}$ power supply. The components necessary to create the experimental circulatory system are listed in Table 1.

The main command unit of the submersible is the ESP WROOM-02 programmable module. The ESP module connects the Inertial Measurement Unit BNO055 from Bosch SensorTech and the direct current motor. Additional information regarding the components used to build the submersible are shown in Table 2. The ESP features integrates a WiFi module 


\begin{tabular}{|c|c|}
\hline Component & Description \\
\hline Tee elbow with PVC door & $\begin{array}{c}\text { Used as immersion and submersion } \\
\text { points, } 2 \text { pieces }\end{array}$ \\
\hline Small tube & $\begin{array}{c}\text { Polyurethane with metal insertions } \\
R=51 / 1000(\mathrm{~m}), h=0.9 / 1000(\mathrm{~m}), \kappa=0.1 .\end{array}$ \\
\hline Large tube & $\begin{aligned} & \text { Polyurethane with metal insertions } \\
R= & 80 / 1000(\mathrm{~m}), h=0.9 / 1000(\mathrm{~m}), \kappa=0.9\end{aligned}$ \\
\hline Funnel & $\begin{aligned} \text { Narrow diameter } d_{n} & =0.102(\mathrm{~m}), \\
\text { Wide diameter } d_{w} & =0.16(\mathrm{~m})\end{aligned}$ \\
\hline Tube & Tube to recirculate the fluid, any diameter \\
\hline Plumbing elbow & Connection purpose, 2 pieces \\
\hline CM10P7-1-24 & Variable flow pump \\
\hline EM-174A & Pump driver \\
\hline SNS-FLOW201 & Flowmeter \\
\hline NI myRIO & Real-time microcontroller \\
\hline Power Supply & 24 (V) supply \\
\hline Non-Newtonian fluid & $\begin{array}{l}\text { Approx. } 10 \text { (l) e.g. water and starch mixtures, } \\
\text { conditioning shampoo, liquid detergent }\end{array}$ \\
\hline
\end{tabular}

TABLE 1. Circulatory system components

\begin{tabular}{|c|c|}
\hline Component & Description \\
\hline Submersible case & The custom hull, 2 halves, 3D printed \\
\hline CRC232 & Battery powering the submersible \\
\hline TPS61090 & $\begin{array}{c}\text { Power booster circuit that ensures the } \\
\text { nominal voltage of }[+2.7,3.0] \mathrm{V}\end{array}$ \\
\hline ESP232 WROOM-02 & WiFi module and microcontroller \\
\hline Bosch BNO055 & $\begin{array}{c}\text { Inertial Measurement Unit - Accelerometer, } \\
\text { Gyroscope and Magnetometer }\end{array}$ \\
\hline Graupner 3 blade propeller & $\begin{array}{c}\text { Propeller for miniature ship design } \\
\text { used to thrust the submersible }\end{array}$ \\
\hline DC motor & 10 mm x 6 mm DC motor \\
\hline DRV8833 & Motor driver \\
\hline Screws & Gelf locking screws, 3 pieces \\
\hline Glue & Gasket sealant, 1 tube \\
\hline
\end{tabular}

TABLE 2. Submersible components 
used to communicate with a server in a bidirectional manner. The submersible sends velocity and positioning data to the server with the purpose of real-time tracking.

For the particular tests in the project to which this work relates, the setup has been designed to consist of two parts: i) a resemblance to the circulatory system and ii) the suspended object, i.e. a submersible capable of navigating through the non-Newtonian liquid. Hence, pulsatile flow is used, in transitional dimensional tubes to obtain pressure gradient variations. The setup is an air-tight circuit.

A snapshot view is presented in Fig. 2. The following protocol is proposed for motion tests. The object is inserted into the system via an immersion point, navigates through the mimicked vein tube and passes the diameter change between the vein and the artery. Subsequently, it travels through the artery mimicked tube and ultimately, it is extracted through the extraction point into a reservoir tank. The liquid flowing through the pipes is a liquid detergent with characteristics similar to the blood such as density and non-Newtonian flow profiles.

The tubes used for mimicking the blood vessels are made of polyurethane with metal insertions, making them slightly flexible. The small tube, resembling a vein, has radius of $R=51 / 1000(\mathrm{~m})$, wall thickness of $h=$ $0.9 / 1000(\mathrm{~m})$ and a ratio of soft-to-hard rings denoted by $\kappa=0.1$. For the larger tube, resembling the artery, the parameters are $R=80 / 1000$ $(\mathrm{m}), h=0.9 / 1000(\mathrm{~m})$ and $\kappa=0.9$. The density of the steel rings is $\rho_{\text {cart }}=1.14 * 1000 \mathrm{~kg} / \mathrm{m}^{3}$ and of the polyurethane $\rho_{\text {soft }}=1.06 * 1000 \mathrm{~kg} / \mathrm{m}^{3}$. The polyurethane elastic modulus is $E s=2.5 * 10^{9}$, while the steel has an elastic modulus of $E c=209 * 10^{9}$.

The pumping of the heart to obtain pulsatile flow profiles is simulated through a variable flow pump, that steers the liquid through veins following a pre-defined a sinusoidal pattern. The viscosity of the liquid detergent mimicking blood is $\mu=0.085 * 10^{-5} \mathrm{~kg} / \mathrm{ms}$, at the pulsing frequency $\omega=$ $2 * \pi * 70 / 60 \mathrm{rad} / \mathrm{s}$ and a density of $\rho=1.03 / 1000 \mathrm{~kg} / \mathrm{m}^{3}$.

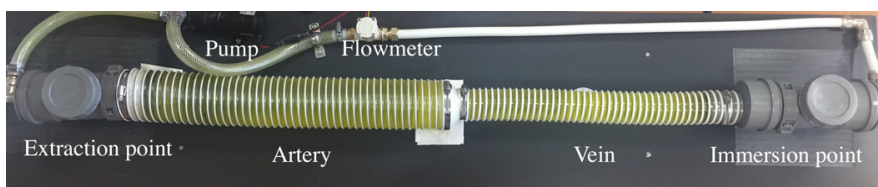

Figure 2. Proposed experimental setup to mimic sections of the circulatory system with non-Newtonian fluid (i.e. liquid detergent) flowing in an air-tight circuit. 
The object is a robot-like submersible, as presented in Fig. 3. The robot is $50 \mathrm{~mm}$ long and $30 \mathrm{~mm}$ wide, with embedded dedicated electronics. The object has been custom designed and the hull was 3D printed to encapsulate a microcontroller, a battery, a WiFi module and an Inertial Measurement Unit. The propeller is actuated by a continuous current motor to which a voltage signal is applied. The propeller generates a thrust allowing the object to move through the non-Newtonian fluid. The velocity of the submersible depends on the surge generated with the propeller and on the interaction with environment. The propeller can rotate in both directions, allowing accelerating, decelerating and standstill motions in pulsatile fluid flow conditions.

An IMU board on the robot enables from the recorded signals to compute the velocity and position, respectively. The well-known problem of the noisy accelerometer data, resulting in an accumulating error that slowly goes towards infinity, has been resolved and presented in [4]. For the current experimental protocol, accurate velocity and positioning data is available.

Further on, in order to closed loop control the velocity and positioning of the submersible, a compensating error law can be implemented in discrete polynomial form by programming the robot. The feedback signal contains the velocity/positioning information obtained from the IMU unit, while the controller output is the voltage given to the DC motor for actuating the propeller's rotation direction and angular velocity.
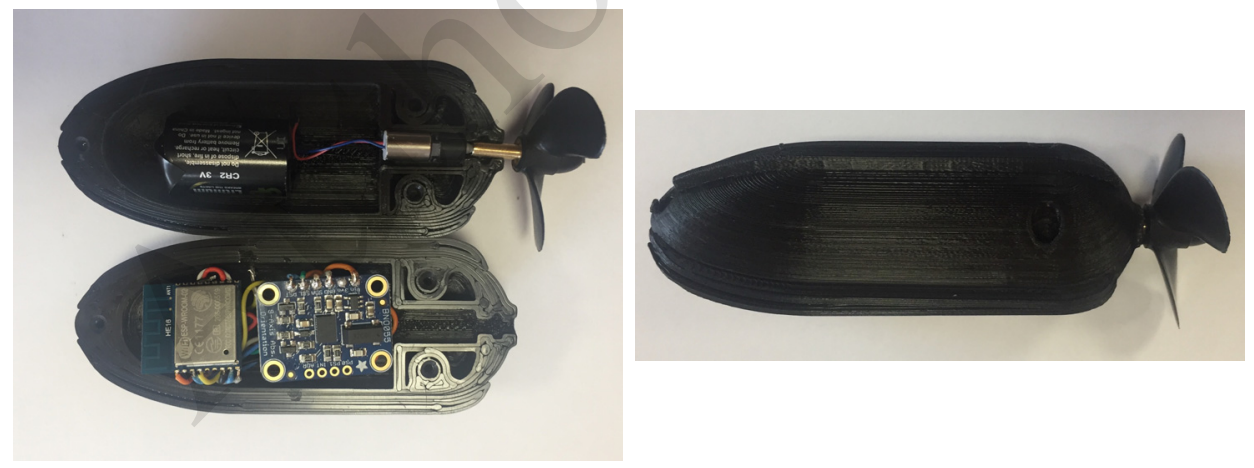

Figure 3. A snapshot view of the submersible robot custom made for this project.

2.5. Considerations on identification for control. For control purposes, a general model is needed that connects the input voltage to the velocity of the submersible. Since the width of the robot is relatively large with respect to the diameter of the tubes, the movement is modeled solely on the longitudinal $X$ axis, i.e. ignoring the heave and sway movements. 
The obtained model has been previously reported in [5], indicating that a fractional order model outperforms the integer order model. This is in accordance to the theoretical concepts presented in here, in a generalized sense of velocity gradient profiles.

The thrust generated by the rotation of the propeller is dependent on several features such as the operating conditions of the propeller (e.g. the liquid in which it is immersed, propeller angular velocity) and the manufacturing characteristics (e.g. rotor blade area, radius, thrust and drag coefficients) 21. The surge velocity of the submersible through the liquid is given by the carefully chosen design parameters of the hull. Here, the important features which will influence the motion are the frontal area, the diameter and the ellipsoid shape of a transverse section, hydrostatic coefficients and input thrust.

In this paper, two approaches are reported. Based on the theoretical concepts generalizing velocity gradient profiles and our prior results, a fractional order (impedance) model has been proposed to be identified on both the analytical and the experimental step response data.

For the identification algorithm, an optimization approach is chosen that minimizes the integral absolute error defined as

$$
\min \int_{0}^{\infty}\left|u_{m}(t)-u(t)\right| d x
$$

where $u_{m}$ is the measured velocity data at time $t$ and $u(t)$ is the step response data at moment $t$ of the system to be identified. The entire fractional-order identification is translated into a classical minimization problem. The optimization is started from an initial transfer function of the form

$$
H_{F O I M}=\frac{b_{m} s^{\beta_{m}}+b_{m-1} s^{\beta_{m-1}}+\ldots+b_{0} s^{\beta_{0}}}{a_{n} s^{\alpha_{n}}+a_{n-1} s^{\alpha_{n-1}}+\ldots+a_{0} s^{\alpha_{0}}},
$$

where parameters $a, b, \alpha$ and $\beta$ are optimized such that the integral absolute error condition is minimized. An initial point must be provided and the obtained results are strongly influenced by the initial conditions. Constraints on the parameter values, on the tolerance interval, as well as on the fractional orders are imposed to reduce the computation time. An iterative procedure is employed to avoid local minimum solutions.

\section{Results and discussion}

3.1. Velocity profiles. Relative axial velocity in the two tubes has been calculated for the relative pressure gradient across the section of the tube and normalized ration of the radial position with respect to the axis of the tube. Fig. 4 (A) indicates the results. 


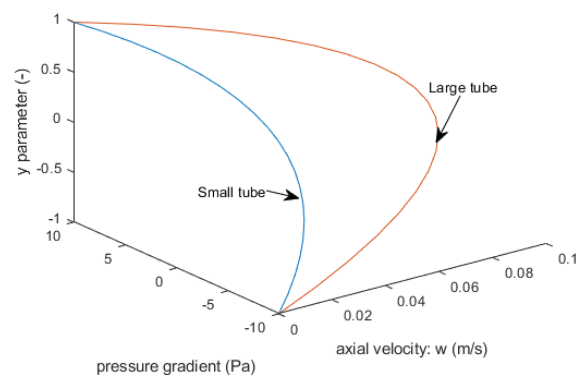

(A)

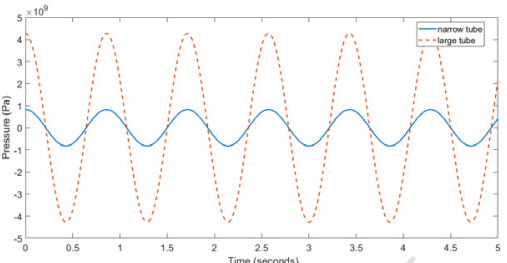

(в)

Figure 4. (A) Velocity profiles in the two tubes (B) Pressure gradient in the two tubes for pulsatile flow.

Damping is decreased by $40 \%$ when going from narrow tube to large tube, thus a relative larger displacement can be expected. This is verified in Fig. 4 (B), where the pressure variations in narrow tube (blue color) is smaller in relative amplitude than the pressure variations in the larger tube (red color). Given the same flow in both tubes, the velocity gradient is expected to be accelerated in the narrow tube. This is in accordance to physical laws and the next step to verify is the effect on the radial velocity. This is important for determining the deviation from axial position during movement of liquid, i.e. frictional head loss.

The velocity profiles for non-Newtonian fluid in steady pipe flow for various values of $\alpha$ are given in Fig. 5a,

3.2. Identification results. The relation between the motor voltage and propeller angular velocity is determined by applying a step input of amplitude $2.4 \mathrm{~V}$ applied to the direct motor through PWM signals. For a linear flow of the liquid detergent, a fractional order model is identified on the experimental step response data of the submersible's velocity inside the small tube as

$$
H_{F O I M}=\frac{0.36}{0.005682 s^{1.7263}+0.11031 s^{0.86825}+1} .
$$

The identified fractional model resembles the second order one with fractional orders of 1.7263 and 0.86825 , respectively. The search for a fractional order model started from the second order transfer function with the static gain $k=0.36$. Optimization is performed in Matlab using the fmincon function and the 'active-set' algorithm. The step response of the identified model is compared against the experimental data in Fig. 5b. 
IDENTIFICATION FOR CONTROL OF SUSPENDED ...
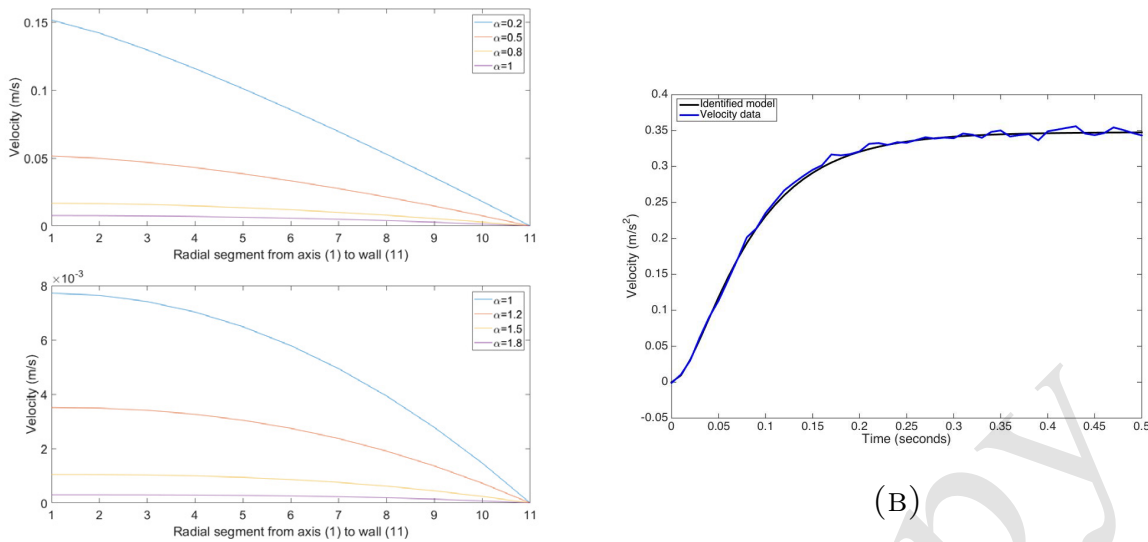

(B)

(A)

Figure 5. (A) Velocity profiles for non-Newtonian fluid based on model (2.17); (B) Comparison of the identified model and the experimental step response data.

3.3. Discussion and limitations. A manifold of applications in specialised literature deal with design, material, manufacturing and functioning of nanorobot objects for motion in non-Newtonian fluids. With that respect, precise, high-performance design and material properties have been investigated over the last decade with exponential success in nanomedicine and other application fields [24, 26]. Our work differentiates to the extent of offering a low-cost, approachable and instructive benchmark solution to the problematic of modelling, identification and control of objects in nonNewtonian fluids.

In this context, the paper succeeded to design, manufacture and implement such a benchmark system, allowing experiments to sustain the theoretical claims. The fractional velocity gradient in developed flow conditions, as well as the dynamic response have been well captured in a non-Newtonian environment, i.e. liquid detergent. Similar reports on such effective benchmarks have not been found in the control engineering literature, hence the work has an original added value to the control community.

An important limitation of the present study refers to the mechanical properties of the tubes selected. Their elasticity is certainly below the biological value of arteries, such that viscoelastic properties are not fully captured. It should be noted that fractional order impedance models have been previously reported to be good candidates for modelling creep and tension, viscoelasticity in arteries [7, 8. Effects of distention and elongation of the tubes during pulsatile flow conditions are not enabled to be 
investigated. The properties of the benchmark, as it is at this moment, are situated somewhere in-between biomedical and technical applications of non-Newtonian fluid mechanics.

Another limitation is perhaps the identification from step response data, a well-known problem 22. The lack of persistent excitation will lead to biased results for high frequency dynamics. Nevertheless, the model seems to capture well enough the steady state gain of the process.

\section{Conclusions}

This paper proposed a theoretical framework and benchmark process to allow generalization of flow dynamics in non-Newtonian fluid environment, i.e. fractional order derivative elements. Given the availability of a laboratory benchmark process and a custom-design submersible object, identification of velocity profile to step response has been performed. The proposed structure is that of a fractional order impedance model, describing the motion of the object in non-Newtonian fluid conditions (i.e. liquid detergent).

Further use of this model may be the controller design for velocity or position control purposes.

\section{Appendix - General Navier-Stokes equations}

The pulsatile flow can be analyzed in terms of periodical functions, such as the pressure gradient: $-\frac{\partial p}{\partial z}=M_{P} \cos \left(\omega t-\Phi_{P}\right)$, where $z$ is the axial coordinate, $\omega=2 \pi f$ is the angular frequency $(\mathrm{rad} / \mathrm{s})$, with $f$ the frequency $(\mathrm{Hz}), M_{P}$ the modulus and $\Phi_{P}$ is the phase angle of the pressure gradient. Given its periodicity, it follows that also the pressure and the velocity components will be periodic, with the same angular frequency $\omega$. The purpose is to determine the velocity in radial direction $u(r, z, t)$ with $r$ the radial coordinate, the velocity in the axial direction $w(r, z, t)$, the pressure $p(r, z, t)$ and to calculate them using the morphological values of the lungs. In this study, we shall make use of the Womersley parameter from the Womersley theory developed for the circulatory system, defined as the dimensionless parameter $\delta=R \sqrt{\frac{\omega \rho}{\mu}}$, with $R$ the absolute radius of the cylinder (pipeline). If the simplest form of axi-symmetrical flow in a cylindrical pipeline is considered, the simplified Navier Stokes equations can be written as $\frac{\partial}{\partial \theta}=\frac{\partial^{2}}{\partial \theta^{2}}=0$ and with the contour velocity $v=0$. Further on, consider no external forces $F_{r}, F_{z}$. For the infinitesimal segment modelled, the total pressure drop variations can be neglected and locally can be divided by density $\rho$ and introduce the dimensionless parameter $y=$ $r / R, 0 \leq y \leq 1$ in the relation $\frac{d}{d y}=\frac{d}{d r} \frac{d r}{d y}=R \frac{d}{d r}, \frac{d}{d r}=\frac{1}{R} \frac{d}{d y}$. The simplifying 
assumptions can be then applied: i) the radial velocity component is small, as well as the ratio $u / R$ and the term in the radial direction; ii) the terms $\frac{\partial^{2}}{\partial z^{2}}$ in the axial direction are negligible, leading to the following system:

$$
\begin{gathered}
\frac{\partial u}{\partial t}=-\frac{1}{\rho R} \frac{\partial p}{\partial y}+\frac{\mu}{\rho}\left[\frac{1}{y R^{2}} \frac{\partial u}{\partial y}+\frac{1}{R^{2}} \frac{\partial^{2} u}{\partial y^{2}}-\frac{u}{R^{2} y^{2}}\right], \\
\frac{\partial w}{\partial t}=-\frac{1}{\rho} \frac{\partial p}{\partial z}+\frac{\mu}{\rho}\left[\frac{1}{y R^{2}} \frac{\partial w}{\partial y}+\frac{1}{R^{2}} \frac{\partial^{2} w}{\partial y^{2}}\right], \\
\frac{u}{R y}+\frac{1}{R} \frac{\partial u}{\partial y}+\frac{\partial w}{\partial z}=0 .
\end{gathered}
$$

Given the pressure gradient is periodic, it follows that also the pressure $p(y, z, t)$ and the other velocity components $u(y, z, t), w(y, z, t)$ are periodic, as in:

$$
\begin{gathered}
p(y, z, t)=A_{P}(y) e^{j \omega(t-z / \tilde{c})}, \\
u(y, z, t)=A_{U}(y) e^{j \omega(t-z / \tilde{c})}, \\
w(y, z, t)=A_{W}(y) e^{j \omega(t-z / \tilde{c})},
\end{gathered}
$$

where $\tilde{c}$ denotes the complex velocity of wave propagation and $j=\sqrt{-1}$. Further simplifications lead to the following system of equations:

$$
\begin{gathered}
u=\frac{j \omega R}{\mu \tilde{c}}\left\{C_{1} \frac{2}{\delta j^{3 / 2}} J_{1}\left(\delta j^{3 / 2} y\right)+\frac{A_{P}}{\rho \tilde{c}} y\right\} e^{j \omega\left(t-\frac{z}{\tilde{c}}\right)}, \\
w=\left\{C_{1} J_{0}\left(\delta j^{3 / 2} y\right)+\frac{A_{P}}{\rho \tilde{c}}\right\} e^{j \omega\left(t-\frac{z}{\tilde{c}}\right)}, \\
p(t)=A_{P} e^{j \omega\left(t-\frac{z}{\tilde{c}}\right)},
\end{gathered}
$$

with $C_{1}=-\frac{A_{P}}{\rho \tilde{c}} \frac{1}{J_{0}\left(\delta j^{3 / 2}\right)}, A_{P}$ the amplitude of the pressure wave, $J_{0}$ the Bessel function of the first kind and zero degree, $J_{1}$ the Bessel function of the first kind and first degree, and in which:

such that

$$
-\frac{d p}{d z}=\frac{j \omega}{\tilde{c}} A_{P} e^{j \omega\left(t-\frac{z}{\tilde{c}}\right)}=M_{P} e^{j\left(\omega t-\Phi_{P}\right)}
$$

$$
A_{P} e^{j \omega\left(t-\frac{z}{\tilde{c}}\right)}=\frac{\tilde{c}}{\omega} M_{P} e^{j\left(\omega t-\Phi_{P}-\pi / 2\right)} .
$$

In this setup, the effective elastic modulus and wall density, respectively, may be considered in function of the pipeline rugged structure:

$$
\begin{aligned}
& E=\kappa E_{c}+(1-\kappa) E_{s}, \\
& \rho_{\text {wall }}=\kappa \rho_{c}+(1-\kappa) \rho_{s},
\end{aligned}
$$

taking into account at each section of the setup the fraction amount $\kappa$ of corresponding hard material (index $c$ ) and soft material (index $s$ ). 


\section{Acknowledgements}

This work was supported by a grant of the Romanian National Authority for Scientific Research and Innovation, CNCS/CCCDI-UEFISCDI, Project number PN-II-P2- 2.1-PED-2016-0101, with PNCDI III.

This work was also supported by the Research Foundation Flanders (FWO) under Grant number 1S04719N.

Dana Copot's contribution is based upon work from COST Action CA15225, a network supported by COST (European Cooperation in Science and Technology).

\section{References}

[1] G. Ala, M. Di Paola, E. Francomano, Y. Li, F. Pinnola, Electrical analogous in viscoelasticity. Commun. Nonlinear Sci. Numer. Simul. 19 (2014), 2513-2527; DOI: 10.1016/j.cnsns.2013.11.007.

[2] A. Berglund, J.A. Liddle, Simultaneous positioning and orientation of a single nano-object by flow control: theory and simulations. New J. of Phys. 13 (2011); DOI: 10.1063/1.2148627.

[3] D. Yin, H. Wu, C. Cheng, Y. Q. Chen, Fractional order constitutive model of geomaterials under thecondition of triaxial test. Intern. J. for Numer. and Anal. Methods in Geomechanics 37, No 8 (2013), 961-972; DOI: 10.1002/nag.2139.

[4] I. Birs, C. Muresan, S. Folea, O. Prodan, An experimental nanomedical platform for controller validation on targeted drug delivery. Proc. of the Australian and New Zealand Control Conf., ANZCC (2017), Gold Coast, Australia; DOI: 10.1109/ANZCC.2017.8298504.

[5] I. Birs, C. Muresan, O. Prodan, S. Folea, C.M. Ionescu, Analytical modeling and preliminary fractional order velocity control of a small scale submersible. 2018 SICE Intern. Symp. on Control Systems, SICEISCS, Japan (2018), 157-162; DOI: 10.23919/SICEISCS.2018.8330170.

[6] D. Copot, R. Magin, R. De Keyser, C.M. Ionescu, Data-driven modelling of drug tissue trapping using anomalous kinetics. Chaos Solitons and Fractals 102 (2017), 441-446; DOI: 10.1016/j.chaos.2017.03.031.

[7] D. Craiem, R. Armentano, A fractional derivative model to describe arterial viscoelasticity. Biorheology 44, No 4 (2007), 251-263; DOI: 10.1109/IEMBS.2006.259709.

[8] D. Craiem, F. Rojo, J. Atienza, R. Armentano, G. Guinea, Fractionalorder viscoelasticity applied to describe uniaxial stress relaxation of human arteries. Phys. Med. Biol. 53, No 17 (2008), 4543-4554; DOI:10.1088/0031-9155/53/17/006.

[9] K. Dekemele, C.M. Ionescu, M. De Doncker, R. De Keyser, Closed loop control of an electromagnetic stirrer in the continuous casting process. 
Proc. of the European Control Conf. (2016), Aalborg, Denmark, 61-66; DOI: 10.1109/ECC.2016.7810264.

[10] A. Dokoumetzidis, R. Magin, P. Macheras, Fractional kinetics in multicompartmental systems. J. Pharmacokinet Pharmacodyn 37 (2010), 507-524; DOI: 10.1007/s10928-010-9170-4

[11] C.M. Ionescu, A memory-based model for blood viscozity. Commun. on Nonlin. Sci. and Numer. Simul. 45 (2017), 29-34; DOI: 10.1016/j.cnsns.2016.09.017.

[12] C.M. Ionescu, J.F. Kelly, Fractional calculus for respiratory mechanics: power law impedance, viscoelasticity and tissue heterogeneity. Chaos, Solitons and Fractals 102 (2017), 433-440; DOI: 10.1016/j.chaos.2017.03.054.

[13] C.M. Ionescu, A. Lopes, D. Copot, J.A.T. Machado, J.H.T. Bates, The role of fractional calculus in modelling biological phenomena: a review. Commun. in Nonlin. Sci. and Numer. Simul. 51 (2017), 141-159; DOI: 10.1016/j.cnsns.2017.04.001.

[14] C.M. Ionescu, J.A.T. Machado, R. De Keyser, Modeling of the lung impedance using a fractional order ladder network with constant phase elements. IEEE Trans. Biomed. Eng. 5, No 1 (2011), 83-89; DOI: 10.1109/TBCAS.2010.2077636.

[15] J.F. Kelly, R.J. McGough, Fractal ladder models and power law wave equations. J. of the Acoustical Soc. of America 126, No 4 (2009), 20722081; DOI: 10.1121/1.4783441.

[16] C.D. Langevin, Modeling axisymmetric flow and transport. Ground Water 46, No 4 (2008), 579-590.

[17] L.G. Leal, The motion of small particles in non-Newtonian fluids. J. of Non-Newtonian Fluid Mechanics 5 (1979), 33-78; DOI: 10.1016/03770257(79)85004-1.

[18] Y. Li, Y.Q. Chen, I. Podlubny, Mittag - Leffler stability of fractional order nonlinear dynamic systems. Automatica 45, No 8 (2009), 19651969; DOI: 10.1016/j.camwa.2009.08.019.

[19] Y. Li, Y.Q. Chen, I. Podlubny, Stability of fractional-order nonlinear dynamic systems: Lyapunov direct method and generalized Mittag-Leffler stability. Computers and Math. with Appl. 59, No 5 (2010), 1810-1821; DOI: 10.1016/j.camwa.2009.08.019.

[20] R. Magin, Fractional calculus in bioengineering. Crit. Rev. Biomed. Eng. 32, No 1 (2004), 1-104; DOI: 10.1615/CritRevBiomedEng.v32.10.

[21] MAN Diesel \& Turbo, Basic Principles of Ship Propulsion (2012).

[22] R. Pintelon, J. Schoukens, System Identification: A Frequency Domain Approach. Wiley-IEEE Press (2001); DOI:10.1002/9781118287422. 
[23] I. Podlubny, Geometric and physical interpretation of fractional integration and fractional differentiation. Fract. Calc. Appl. Anal. 5, No 4 (2002), 367-386; see at: arXiv:math/0110241.

[24] Y. Saadeh, D. Vyas, Nanorobotic applications in medicine: current proposals and designs. Amer. J. Robot. Surg. 1, No 1 (2014), 4-11; DOI: $10.1166 /$ ajrs.2014.1010.

[25] H. Schiessel, A. Blumen, Mesoscopic pictures of the sol-gel transition: ladder models and fractal networks. Mectromolecules 28 (1995), 40134019; DOI: 10.1021/ma00115a038.

[26] J. Venugopal, M.P. Prabhakaran, S. Low, A.T. Choon, Y.Z. Zhang, G. Deepika, S. Ramakrishna, Nanotechnology for nanomedicine and delivery of drugs. Curr. Pharm. Des. 14, No 8 (2008), 2184-2200; DOI: 10.2174/138161208785740180.

[27] B. West, Fractal physiology and the fractional calculus: a perspective. Frontiers in Physiology (2010); DOI: 10.3389/fphys.2010.00012.

[28] C. Ionescu, A. Lopes, D. Copot, J.A.T. Machado, J.H.T. Bates, The role of fractional calculus in modeling biological phenomena: A review. Commun. in Nonl. Sci. and Numer. Simul. 51 (2017), 141-159; DOI: 10.1016/j.cnsns.2017.04.001.

[29] D. Yin, W. Zhang, C. Cheng, L. Yi, Fractional time-dependent Bingham model for muddy clay. J. Non-Newtonian Fluid Mech. 187 (2012), 32-35; DOI: 10.1016/j.jnnfm.2012.09.003.

1 Technical University of Cluj-Napoca, Department of Automation Memorandumului Str., No. 28, Cluj Napoca, ROMANIA

e-mails: Isabela.Birs@aut.utcluj.ro Cristina.Muresan@aut.utcluj.ro , Ioan.Nascu@aut.utcluj.ro Revised: July 26, 2019

${ }^{2}$ Ghent University, Faculty of Engineering and Architecture Department of Electrical Energy, Systems and Automation Technologiepark 914, 2nd Floor, 9052 - Ghent, BELGIUM

${ }^{3}$ EEDT Group, Member of Flanders Make consortium, BELGIUM e-mails:Dana.Copot@ugent.be , ClaraMihaela.Ionescu@ugent.be

Please cite to this paper as published in:

Fract. Calc. Appl. Anal., Vol. 22, No 5 (2019), pp. 1378-1394, DOI: 10.1515/fca-2019-0072; at https://www.degruyter.com/view/j/fca. 